\title{
Role of myosin light chain kinase in intestinal epithelial barrier defects in a rat model of bowel obstruction
}

\author{
Chi-Chin Wu, Yen-Zhen Lu, Li-Ling Wu and Linda C Yu*
}

\begin{abstract}
Background: Bowel obstruction is a common cause of abdominal emergency, since the patients are at increased risk of septicemia resulting in high mortality rate. While the compartmentalized changes in enteric microfloral population and augmentation of bacterial translocation (BT) have already been reported using experimental obstruction models, alterations in epithelial permeability of the obstructed guts has not been studied in detail. Myosin light chain kinase (MLCK) is actively involved in the contraction of epithelial perijunctional actinomyosin ring and thereby increases paracellular permeability. In the current study we attempt to investigate the role of MLCK in epithelial barrier defects using a rat model of simple mechanical obstruction.
\end{abstract}

Methods: Wistar rats received intraperitoneal injection of ML-7 (a MLCK inhibitor) or vehicle at 24, 12 and 1 hrs before and $12 \mathrm{hrs}$ after intestinal obstruction (IO). The distal small intestine was obstructed with a single ligature placed $10 \mathrm{~cm}$ proximal to the ileocecal junction in 10 rats for 24 hrs. Sham-operated rats served as controls.

Results: Mucosal injury, such as villous blunting and increased crypt/villus ratio, was observed in the distal small intestine of $I O$ rats. Despite massive enterocyte shedding, intestinal villi were covered with a contiguous epithelial layer without cell apoptosis. Increased transmural macromolecular flux was noticed in the distal small intestine and the proximal colon after $\mathrm{IO}$. The bacterial colony forming units in the spleen and liver of $\mathrm{IO}$ rats were significantly higher than those of sham controls. Addition of ML-7 ameliorated the IO-triggered epithelial MLC phosphorylation, mucosal injury and macromolecular flux, but not the level of BT.

Conclusions: The results suggest that $1 \mathrm{O}$-induced premature enterocytic sloughing and enhanced paracellular antigenic flux were mediated by epithelial MLCK activation. In addition, enteric bacteria may undergo transcytotic routes other than paracellular paths to cross the epithelium.

\section{Background}

Bowel obstruction is one of the most common causes of abdominal emergency. Single or multiple sites of obstructions are found in patients with post-operative adhesions, incarcerated hernia, gut strangulation, intussusception, and abdominal tumors [1-3]. Patients with mechanical bowel obstruction are of increased risk of septicemia which results in high rates of mortality $[1,2]$. The septic complications are associated with gut barrier dysfunc-

* Correspondence: Ichyu@ntu.edu.tw

1 Graduate Institute of Physiology, National Taiwan University College of Medicine, Taipei, Taiwan

Full list of author information is available at the end of the article tion, and entry of gut microorganisms and bacterial products $[4,5]$.

Intestinal lumen hosts a large amount of commensal bacteria that is normally separated from the body proper by epithelial cells linked by tight junctions [6,7]. The highest number of bacteria is found in the colon with a gradual decrease in upper bowel segments. In obstructed guts, massive quantity of bacteria and antigens penetrates into extraintestinal organs, which is termed bacterial translocation (BT) $[4,5,8-10]$. Changes in gut microfloral population have been implicated in the mechanism of BT. In a murine model of single-site bowel obstruction, the bacterial population in various gut segments multiplied 
100 to 1000 folds [8]. Interestingly, bacterial overgrowth was more pronounced in the ileum compared to the cecum or colon [8]. Aside from the phenomena of BT and compartmentalized bacterial overgrowth, limited information exists on changes of epithelial permeability in obstructed guts.

The epithelial tight junctions (TJ) are regulated by the contraction of perijunctional actinomyosin ring. The phosphorylation of myosin light chain (MLC) by myosin light chain kinase (MLCK) results in increased paracellular permeability [11-13]. The phenomena of MLC phosphorylation and $\mathrm{TJ}$ reorganization are also involved in epithelial shedding at villous tips, contributing to normal cell turnover along the crypt-villus axis [14]. Physiological extrusion of enterocytes does not compromise the epithelial barrier function. However, a rise in intestinal permeability caused by MLCK activation was demonstrated in numerous disease models, including endotoxemia, pro-inflammatory stress, giardiasis and $\mathrm{T}$ cellactivated colitis [15-17]. Whether bowel obstruction could induce antigenic flux and BT via activation of epithelial MLCK has not been examined so far.

Hence we determined to investigate the compartmentalized changes of epithelial permeability, and to assess the role of MLCK in abnormal epithelial shedding, macromolecular influx and BT in obstructed guts, using a rat model of simple mechanical obstruction.

\section{Methods}

Animals

Male Wistar rats aged 6 to 9 weeks and weighing 200-300 $\mathrm{g}$ were used for the study. Animals were raised in a temperature-controlled room $\left(20 \pm 2^{\circ} \mathrm{C}\right)$ with 12-hr lightdark cycles, and fed with regular rat chow and water. The experimental procedures were approved by the Laboratory Animal Care Committee, National Taiwan University College of Medicine.

\section{Experimental design}

In the first set of experiments, rats were randomly divided to sham-operation (sham), and intestinal obstruction (IO) groups ( $\mathrm{n}=5$-7/group). The experimental protocols were carried out under aseptic conditions. After anesthetizing with sodium pentobarbital $(50 \mathrm{mg} /$ $\mathrm{kg}$, intraperitoneal (i.p.) injection), rats underwent midline laparotomy. In IO rats, the distal small intestine was obstructed with a single ligature of 4-0 silk placed $10 \mathrm{~cm}$ proximal to the ileocecal junction. Sham-operated rats received mock manipulation of the gut without ligation. In all animals, care was taken not to occlude or puncture mesenteric vessels and no sign of cyanosis was seen during the experiment. The abdominal wall was sutured after surgery and the incision sterilized with povidone iodine. During these procedures, the arterial blood pressure, heart rate, and body temperature were closely monitored. The average operating time per animal was less than 15 minutes. The rats were placed into steel cages, fasted but with free access to water. Twenty-four hours later, sham and $\mathrm{IO}$ rats were sacrificed. In addition, ischemia/reperfusion $(\mathrm{I} / \mathrm{R})$ rats were used as positive controls to show epithelial discontinuity and villous denudation. The superior mesenteric artery was occluded with an artery clamp for 20 minutes and released for one hour for reperfusion. The gut tissues were collected at the end of the procedure.

In the next series of experiments, animals were randomized to four experimental groups ( $n=5-7$ /group): group 1 (sham+vehicle); group 2 (IO+vehicle); group 3 (sham+ML-7), and group 4 (IO+ML-7). ML-7 (5iodonaphthalene-1-sulfonyl, Sigma) is a selective MLCK inhibitor. ML-7 $(1 \mathrm{mg} / \mathrm{kg})$ or its vehicle $(0.9 \% \mathrm{NaCl})$ was administered i.p. in a volume of $250 \mu \mathrm{l}$ at 24, 12, and $1 \mathrm{hr}$ before and $12 \mathrm{hrs}$ after intestinal ligation or sham-operation. ML-7 was used at a dose previously described in rodent models to inhibit LPS- and stress-induced increase of intestinal paracellular permeability $[15,16]$. No bactericidal or bacteristatic activity was seen with ML-7 in our preliminary study. The animals received either sham-operation or IO were sacrificed $24 \mathrm{hrs}$ after surgery. Two 2-cm intestinal segments from the duodenum (starting at $1 \mathrm{~cm}$ distal to the pylorus), jejunum (15 $\mathrm{cm}$ distal to the pylorus), distal small intestine $(10 \mathrm{~cm}$ proximal to the ileocecal junction), proximal colon, and distal colon [18] were excised for experiments.

\section{Histopathological examination}

Tissues were fixed in 4\% paraformaldehyde and embedded. Sections of $4 \mu \mathrm{m}$ thickness were stained and examined by light microscopy. The villous height and crypt depth were measured from well-oriented, complete crypt-villous units using a scale built in the optical lens of the microscope. Three-to-five crypt-villus units were measured in each rat sample. The average of the calculated ratio of crypt to villus was determined for each rat in the different groups [19].

Light microscopic studies were reviewed in a blinded manner using a histopathological scale previously described [20]. Briefly, the tissue damage was graded from 0 to 5 according to the following criteria: grade 0 , normal structure of villi; grade 1 , development of small subepithelial space at the villous apex; grade 2, enlarged subepithelial space but without change in villous length and width; grade 3 , few shortened villi and presence of cells in the lumen; grade 4 , the majority of villi are shortened and widened with crypt hyperplasia and cells in the lumen; grade 5, blunting of all villi with elongated crypts and a high number of cells in the lumen. 


\section{TUNEL assay}

DNA-strand breaks (the hallmark of cell apoptosis) were detected in situ by a terminal deoxynucleotide transferase biotin-dUTP nick-end labeling (TUNEL) method. A TdTFragEL ${ }^{\mathrm{TM}}$ DNA fragmentation detection kit (Calbiochem) was utilized following the manufacturer's protocol. The number of TUNEL(+) cells was quantified in photomicrographs of intestines taken from three-to-five rats per group at $100 \times$ magnification.

\section{Ussing chamber studies and Permeability assay}

Intestinal segments were removed and gently rinsed with PBS. Care was taken to avoid Peyer's patches. Musclestripped tissues were opened along the mesenteric border and mounted in Ussing chambers (WPI Instruments). The opening area $\left(2 \mathrm{~cm}^{2}\right)$ of the chamber exposed the tissue to $5 \mathrm{ml}$ of circulating oxygenated Krebs buffer. The serosal buffer contained $10 \mathrm{mmol} / \mathrm{L}$ of glucose that was osmotically balanced with $10 \mathrm{mmol} / \mathrm{L}$ of mannitol in the mucosal buffer. The temperature of the buffer was maintained at $37^{\circ} \mathrm{C}$ using a circulating water bath. The tissues were clamped at $0 \mathrm{~V}$ using a voltage clamp. The potential difference $(\mathrm{PD}, \mathrm{mV})$ and the short-circuit current (Isc, $\left.\mu \mathrm{A} / \mathrm{cm}^{2}\right)$ of the tissues were determined on line. The tissues were pulsed with $1 \mathrm{mV}$ for a duration of one second in 5-minute intervals, and the change in the Isc caused by the pulse was used to calculate the tissue conductance $\left(\mathrm{mS} / \mathrm{cm}^{2}\right)$ according to Ohm's law [21,22].

The intestinal epithelial permeability was determined by the level of mucosal-to-serosal flux of horseradish peroxidase (HRP, type II, MW = $44 \mathrm{kD}$, Sigma). Tissues on the Ussing Chambers were allowed to equilibrate until the Isc stabilized before HRP was added to the luminal buffer at a final concentration of $5 \times 10^{-5} \mathrm{~mol} / \mathrm{L}$. Samples $(300 \mu \mathrm{l})$ of serosal buffer were collected at $0,30,60$ and 90 minutes after luminal addition of HRP, and were replaced with Krebs buffer. The concentration of HRP was determined by a kinetic enzymatic assay. Fluxes were calculated according to standard formulae and were expressed as $\mathrm{pmol} / \mathrm{cm}^{2} / \mathrm{hr}[21,22]$.

\section{Analysis of bacterial translocation (BT)}

Under sterile conditions, parts of the liver and spleen were removed and weighed. Each organ was homogenized and sonicated in 10 times volume of sterile PBS, and cultured on fresh blood agar plates (Scientific Biotech Corp.) at $37^{\circ} \mathrm{C}$ for 24 hours. The bacterial colonies were counted and normalized to colony-forming units per gram of tissue (CFU/g) [23].

\section{Measurement of luminal bacterial counts}

The distal small intestine of $10-\mathrm{cm}$ length, with thread ligation at one end, was removed after IO for $24 \mathrm{hrs}$. The intestinal loop was instilled with sterile PBS $(0.25 \mathrm{ml})$ and rocked back and forth for 10 times. The intestinal lavage was plated on fresh blood agar overnight, and the number of bacteria was expressed as log colony-forming units per $\mathrm{ml}(\log \mathrm{CFU} / \mathrm{ml})$.

\section{Western Immunoblotting of phosphorylated MLC (pMLC)}

Scraped mucosa was homogenized in 10 times volume of complete RIPA buffer and sonicated for 10 seconds. The lysate was centrifuged and the protein concentration of the supernatant was adjusted to $5 \mathrm{mg} / \mathrm{ml}$. The supernatant was dissolved in $2 \times$ electrophoresis sample buffer. Samples were boiled in $95^{\circ} \mathrm{C}$ heat block, and stored at $20^{\circ} \mathrm{C}$ until the use for immunoblotting.

The samples were separated by $4-12 \%$ SDS/PAGE, and the resolved proteins were electrotransferred onto membranes. After blocking in 5\% nonfat dry milk, the membrane was incubated with a polyclonal rabbit anti-human phospho-MLC antibody (1:200, Santa Cruz) or a monoclonal mouse anti- $\beta$-actin $\left(1: 2000\right.$, Sigma) at $4^{\circ} \mathrm{C}$ overnight [16]. Membranes were washed and incubated with goat anti-rabbit IgG (1:1000, Cell Signaling) and goat anti-mouse IgG (1:2000, Santa Cruz) for 1 hour. After additional washes, the antigens were revealed using chemiluminescence (ECL) detection reagents (Millipore). Band density was quantified by photoimage analysis.

\section{Immunofluorescent staining}

Intestinal tissue sections were blocked and incubated with rabbit anti-phospho-myosin light chain 2 (Cell Signaling) overnight at $4^{\circ} \mathrm{C}$. The sections were washed with PBS and incubated with Alexa Fluor 488 goat anti-rabbit IgG (Invitrogen) for $1 \mathrm{hr}$. The sections were washed, and the cell nuclei were stained with a Hoechst dye (Invitrogen). Images were captured using a fluorescence microscope.

\section{ELISA}

Scraped mucosa was homogenized and sonicated in PBS, and the lysate was centrifuged. The protein concentration in the supernatant was quantified. The levels of TNF $\alpha$ in intestinal mucosa and plasma samples were measured by using ELISA development kits (PeproTech) according to the manufacturer's instruction. The cytokine levels in intestinal mucosa were expressed in $\mathrm{pg} / \mathrm{mg}$ of protein.

\section{Statistical analysis}

All data except for bacterial CFU/g were presented as mean \pm SEM. The data were compared by ANOVA, followed with Student-Newman-Keuls post-hoc test wherever appropriate (Sigma Stat). The median of CFU/g values were compared using the Mann Whitney nonparametric test. The villous length, crypt depth, crypt/villi ratio and histological damage score were compared using 
(A)

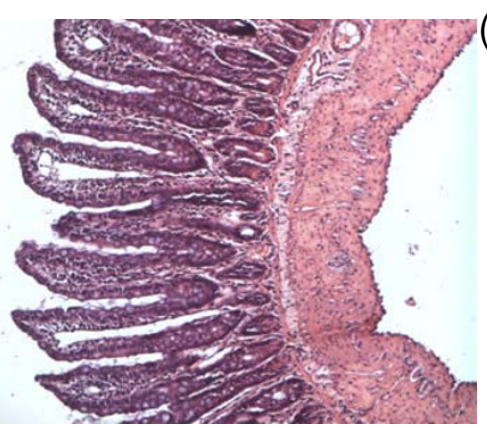

(C)

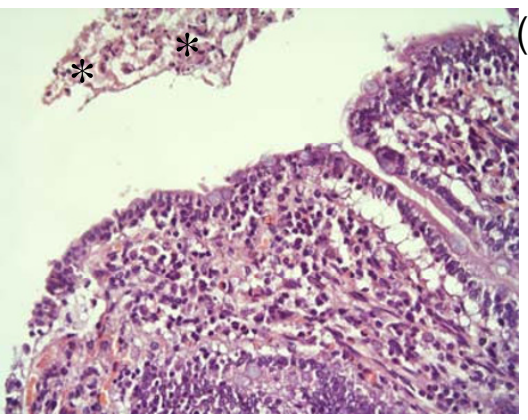

(E)

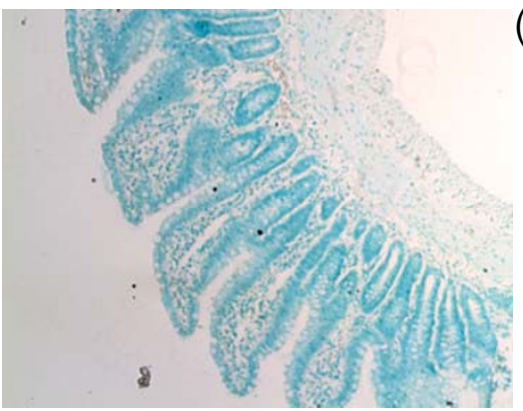

(B)

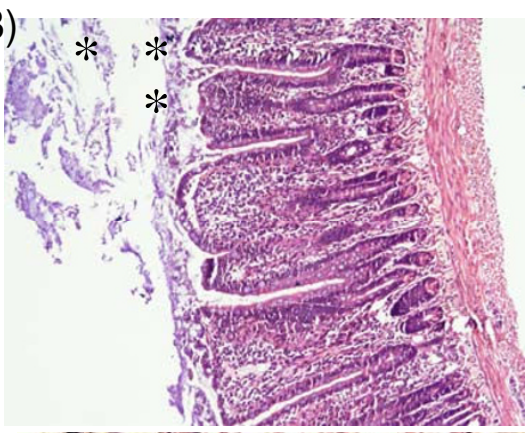

(D)

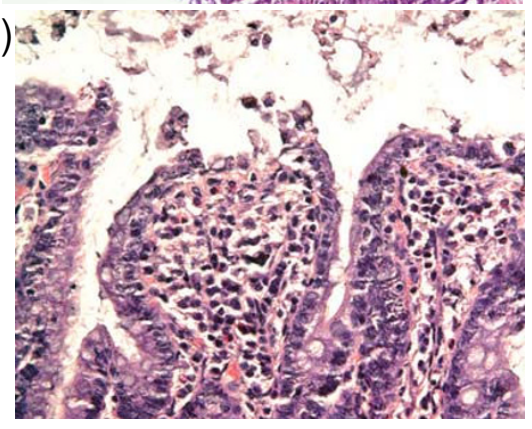

(F)

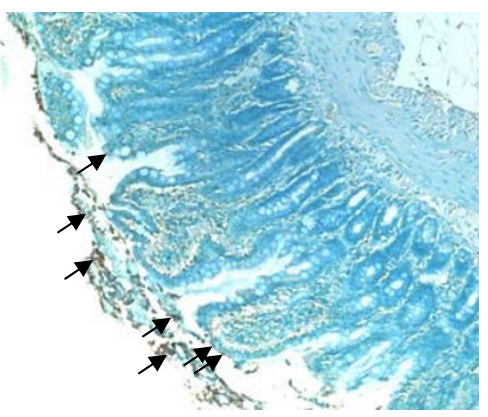

(G)

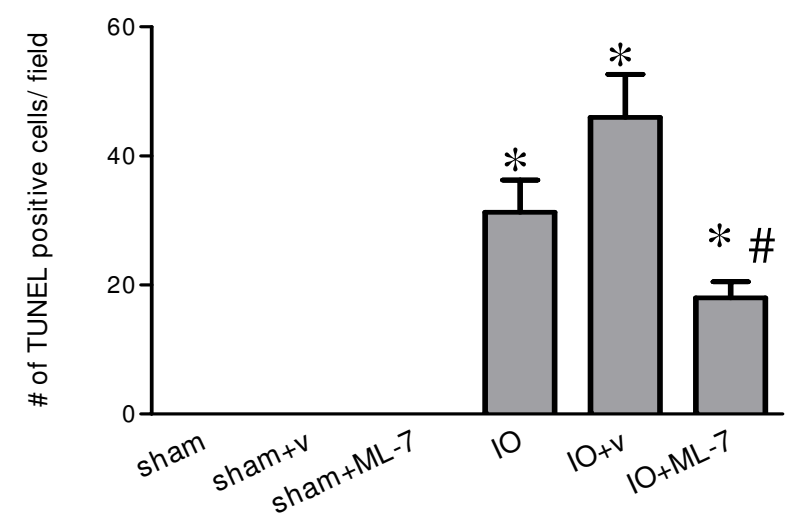

Figure 1 Histological images of the distal small intestine of sham and 10 rats. (Panels A-D) Sections of intestinal tissues were stained with $\mathrm{H}$ \& E. (Panels E and F) The apoptotic level of intestinal tissues was examined by TUNEL assay. (A) Normal villous structure was seen in the distal small intestine of sham rats. (B) Following IO, villi were edematous and blunted. Severe shedding of epithelial cells was observed in the intestinal lumen of $\mathrm{IO}$ rats (asterisk). (C) Higher magnification of the epithelial layer in the distal small intestine of $\mathrm{IO}$ rats showing epithelial shedding without villous denudation. (D) A positive control displaying epithelial discontinuity in an ischemic gut model. (E) No sign of TUNEL(+) cells was observed in the intestine of sham controls. (F) A high number of apoptotic cells was seen in the gut lumen following $I O$ (arrows). (G) Quantification of TUNEL(+) cells. * $\mathrm{p}<$ 0.05 vs. respective sham groups. $\# p<0.05$ vs. IO+v rats. Panels $A, B, E$ and $F: 100 \times$ magnification. Panels C and D: 400x magnification. 
(A)

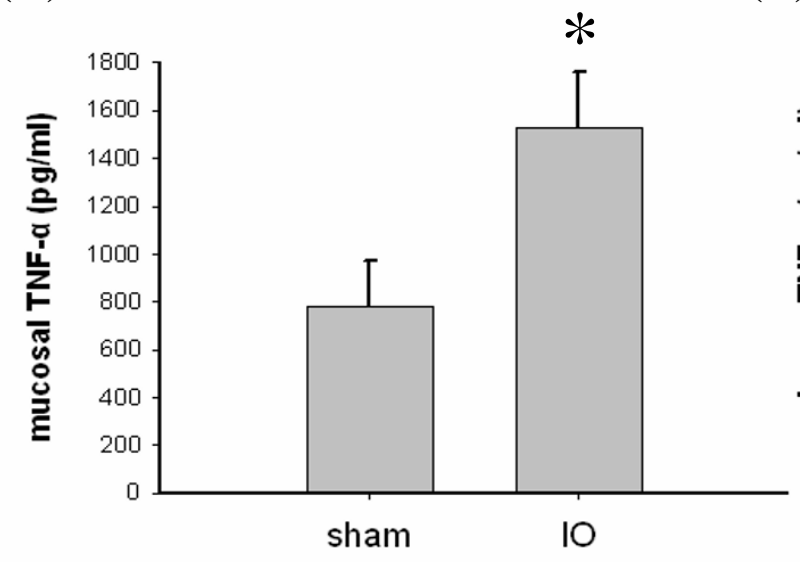

(B)

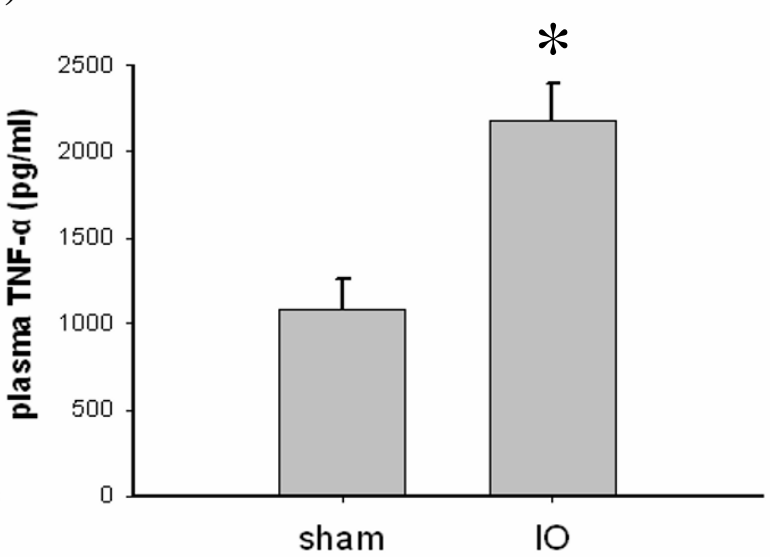

Figure 2 Enhanced mucosal and plasma TNFa levels following IO. (A) The TNFa levels in the mucosa of the distal small intestines in sham and IO rats. (B) The plasma TNFa concentration in sham and 10 rats. $n=5 /$ group. ${ }^{*} p<0.05$ vs. sham rats.

t-test. A value of $\mathrm{p}<0.05$ was considered statistically significant.

\section{Results}

Intestinal obstruction (IO) caused histological injury and inflammation in the distal small intestine

In contrast to the normal villous structure in sham rats (Figure 1A), mucosal injury, such as villous blunting and epithelial sloughing, were observed in the distal small intestine of $\mathrm{IO}$ rats (Figure 1B). The crypt/villi ratio in distal small intestine of sham rats was significantly lower than that of IO rats $(0.651 \pm 0.023$ vs. $1.124 \pm 0.107, \mathrm{p}<$ $0.05)$. The majority of the shortened villi were seen fully covered with a contiguous epithelial layer without surface denudation in IO rats (Figure $1 \mathrm{~B}$ and $1 \mathrm{C}$ ). A positive control showing villous epithelial discontinuity was previously established in ischemic gut models (Figure 1D). Increased cellularity and inflammatory infiltrate were seen in the lamina propria of IO rats. Moreover, the levels of TNF $\alpha$ in the intestinal mucosa and plasma samples of IO rats were higher than those of sham controls (Figure 2).

A higher number of TUNEL (+) apoptotic cells in the intestinal lumen was noticed in $\mathrm{IO}$ rats compared to sham controls (Figure 1E-G). Most apoptotic cells were present in the luminal area but not on the villous surface of IO rats (Figure 1F). The overall histopathological damage score in the intestine of sham and IO rats were statistically different $(0.70 \pm 0.30$ vs. $4.50 \pm 0.16, \mathrm{p}<0.05)$. The mucosal histology and cell apoptosis level in other segments of the intestine, including duodenum, jejunum, proximal and distal colon, were comparable between sham and IO rats (data not shown).
Enhanced transepithelial permeability to ions and macromolecules was found in the distal small intestine and proximal colon after 10

To investigate the effect of IO on epithelial barrier function, gut segments were collected for the measurement of transmural electrical conductance and HRP flux. The conductance of the distal small intestine in IO rats $(81.69$ $\left.\pm 15.23 \mathrm{mS} / \mathrm{cm}^{2}\right)$ was higher than that of sham controls $\left(33.22 \pm 3.74 \mathrm{mS} / \mathrm{cm}^{2}\right)$ (Figure $\left.3 \mathrm{~A}\right)$. While increased tissue conductance was also found in the proximal colon of IO rats, the duodenum, jejunum and distal colon in IO rats were unaffected compared to sham controls (Figure 3A). The transmural flux rate of HRP was significantly higher in the distal small intestine of IO rats compared to their sham counterparts (Figure 3B). A statistically significant rise in HRP flux was also noticed in the proximal colonic tissues in $\mathrm{IO}$ rats (Figure $3 \mathrm{~B}$ ).

\section{IO triggered enteric bacterial overgrowth and bacterial translocation to the spleen and liver}

A tenfold increase of the luminal bacterial counts was seen in the distal small intestine of IO rats compared to sham controls, suggesting enteric bacterial overgrowth following bowel obstruction (Figure 4). A significant increase in the mean bacterial CFU was found in the spleen of IO rats compared to that of sham rats $(672.7 \pm$ 222.3 vs. $10.0 \pm 6.7 \mathrm{CFU} / \mathrm{g}, \mathrm{p}<0.05)$. The amount of viable bacteria in the liver was also higher in IO rats than in sham controls $(300.0 \pm 72.0$ vs. $0.0 \pm 0.0 \mathrm{CFU} / \mathrm{g}, \mathrm{p}<0.05)$. Due to the large variation among bacterial CFU numbers in each group, the median scores were analyzed by nonparametric statistical methods which showed similar results as well. 


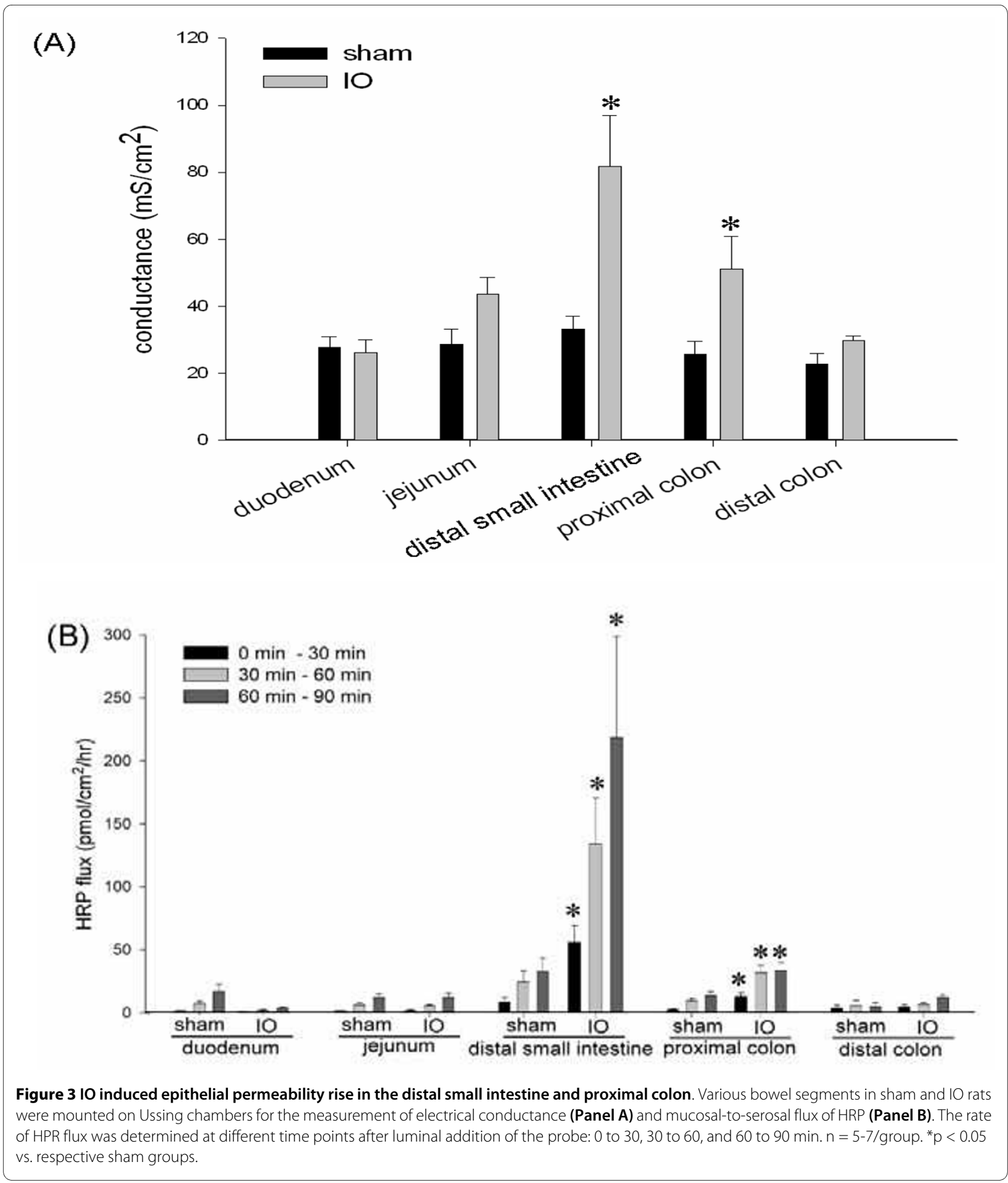

Increased MLC phosphorylation was found in the intestinal epithelium of $\mathrm{IO}$ rats

A 3-fold increase in the level of phosphorylated MLC was seen in the intestinal mucosa of IO rats compared to sham controls (Figure 5A). Immunohistological staining confirmed the increase of pMLC on villous epithelium following $\mathrm{IO}$ (Figure $5 \mathrm{~B}$ and $5 \mathrm{C}$ ). No staining was observed in the villous core or muscular layer in the distal small intestine of IO rats ruling out the involvement of endothelial and smooth muscle cells which also contain MLC. Isotype antibody controls showed negative staining (data not shown). To decipher whether MLCK activation was involved in the intestinal permeability changes, rats were injected (i.p.) with ML-7 (an inhibitor to MLCK) or 


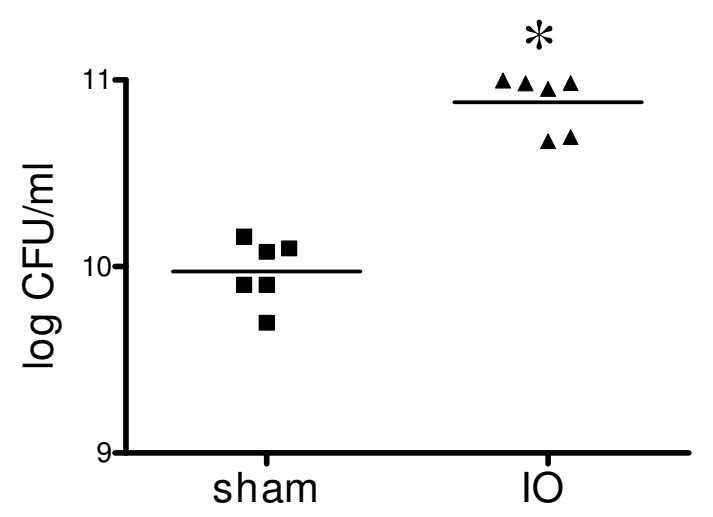

Figure 4 Bacterial overgrowth in the small intestinal lumen in IO rats. A tenfold increase of bacterial colony forming units (CFU) was seen in the intestinal lavage in $1 O$ rats compared to sham controls. Each data point in the figure represents the value from one animal, and the mean values of bacterial counts are shown as bars. $n=6$ /group. ${ }^{*} p<$ 0.05 vs. sham rats.

vehicle (v) before operation. Treatment with ML-7 reduced the increase of pMLC levels in the intestinal epithelium caused by IO (Figure 5A-E).

\section{Treatment with ML-7 alleviated mucosal injury, and prevented the rise of gut permeability in 10 rats}

The IO-induced mucosal injury, including villous blunting and epithelial sloughing, was reduced by the administration of ML-7 (Figure 6A-D). Increased crypt/villi ratio was seen in $\mathrm{IO}+\mathrm{v}$ rats compared to sham $+\mathrm{v}$ rats $(0.970 \pm$ 0.061 vs. $0.667 \pm 0.036, \mathrm{p}<0.05)$, whereas those of $\mathrm{IO}+\mathrm{ML}-7$ and sham $+\mathrm{ML}-7$ rats were comparable $(0.630$ \pm 0.025 vs. $0.637 \pm 0.059, \mathrm{p}>0.05$ ) (Table 1$)$. The number of apoptotic cells in IO+ML-7 rats was significantly lower than that of $\mathrm{IO}+\mathrm{v}$ rats (Figure $1 \mathrm{G}$ and Figure $6 \mathrm{E}-\mathrm{H}$ ). Treatment of ML-7 decreased the overall histopathological score, and prevented the abnormal increase of conductance and HRP flux caused by IO (Figure 7). Vehicle injection had no effect on these parameters.

The mean bacterial CFU in the spleen and liver of $\mathrm{IO}+\mathrm{ML}-7$ rats were slightly lower than that of $\mathrm{IO}+\mathrm{v}$ rats, however, the results did not reach statistical significance (Figure 8). The median scores were also used for nonparametric statistical analysis and showed similar results.

\section{Discussion and conclusions}

Results of the current study demonstrate that bowel obstruction by mechanical ligation evoked mucosal injury and gut barrier damage. The histological damage in our IO rats was similar to other models of mechanical obstruction showing swollen and edematous villous structure [8,24]. The continuity of epithelial layer on the villi was not disrupted in IO rats, in comparison to mod- els with mesenteric ischemia/reperfusion injury that display severe villous denudation and tissue necrosis $[9,23]$. In consistence with a previous study [8], mucosal damages were only present in close vicinity to the obstructed site, whereas no change was discernible in other parts of the intestine. Moreover, elevated ionic and macromolecular permeability were observed in the distal small intestine and proximal colon, but not in other gut segments, of IO rats.

Enteric bacterial overgrowth in the distal small intestine was associated with an increase in viable bacterial count in the spleen and liver in IO rats. The phenomenon of enhanced BT to extraintestinal organs is consistent with previous reports [8-10]. The most common bacterial species identified in the mesenteric lymph nodes, liver, spleen, and blood samples in bowel obstruction were Escherichia coli, Klebsiella sp., Bacteroids, Proteus mirabilis, Enterococcus sp. $[4,8]$. Results from animal models and patient studies indicated that enteric bacterial overgrowth and microfloral ecological changes may contribute to the influx of enteric bacteria $[4,8,25]$. Abnormal epithelial permeability and downregulated immunity also played crucial roles in the mechanism of enhanced BT $[5,16,26]$. We and others showed that IO induced compartmentalized changes in mucosal histology, epithelial permeability and bacterial overgrowth, confined to the distal small intestine. These findings suggested that the distal small intestine could be the major site of bacterial and antigenic influx. Hence we further explored the molecular mechanism responsible for these pathological phenomena.

Excessive epithelial cell apoptosis in situ has been suggested to be a causative factor for cell detachment and barrier dysfunction [27-29]. Previous studies demonstrated that inhibition of caspase activation attenuated the increase of epithelial permeability in rodent intestinal transplantation models and in human intestinal cell cultures [29-32]. Our study showed low numbers of apoptotic cells on the villous surface of IO rats and thus, ruled out the possibility that excessive villous apoptotic cell death triggered the enhanced epithelial permeability. The apoptotic cells were found in the intestinal lumen, indicating that IO elicits severe villous epithelial sloughing in which the enterocytes undergo cell death after detachment.

Previous cell culture studies demonstrated that proinflammatory cytokines, e.g. TNF $\alpha$ and IFN $\gamma$, disrupted tight junctional structures and upregulated MLCK expression, leading to increase of epithelial permeability $[33,34]$. We showed that the level of intestinal mucosal TNF $\alpha$ was elevated following IO, which may partly contribute to the induction of intestinal barrier damage. The pharmacological agent (ML-7) was used to decipher the role of MLCK, since ML-7 exerts its inhibitory effect on 


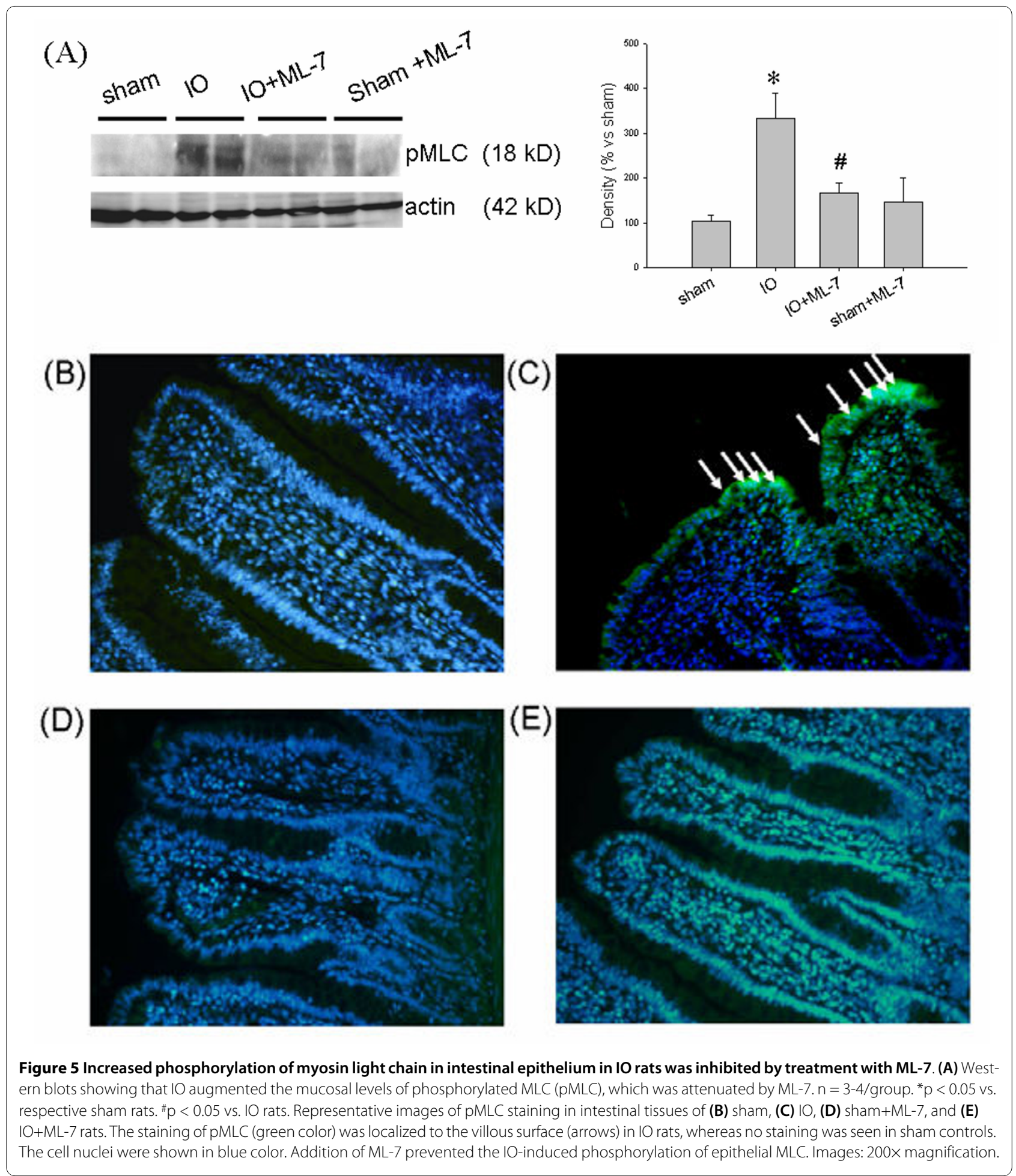

both isoforms of MLCK by blocking the ATP binding sites on the kinase. MLCK consists of short $(130 \mathrm{kD})$ and long $(220 \mathrm{kD})$ forms. The long form accounts for $\sim 97 \%$ of MLCK activity in the intestinal epithelial cells, whereas short MLCK is mainly involved in smooth muscle con- traction $[35,36]$. We confirmed that the phosphorylation level of epithelial MLC was elevated after IO. Addition of ML-7 significantly reduced the epithelial pMLC level in IO rats. The IO-induced rise of tissue conductance and HRP flux were abolished by treatment with ML-7, sug- 
(A)

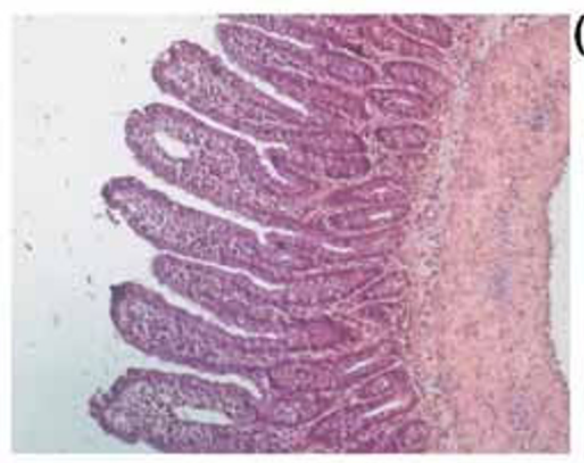

(C)

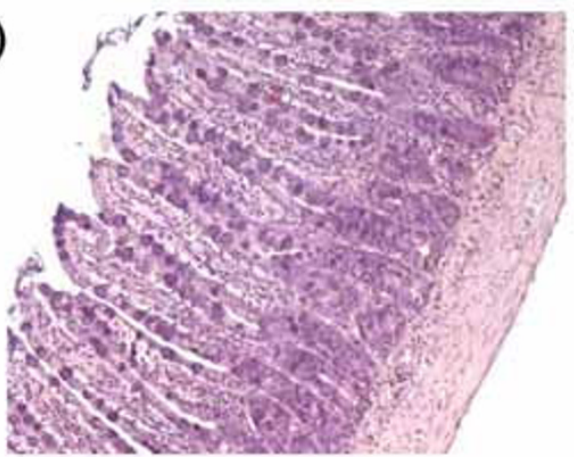

(E)

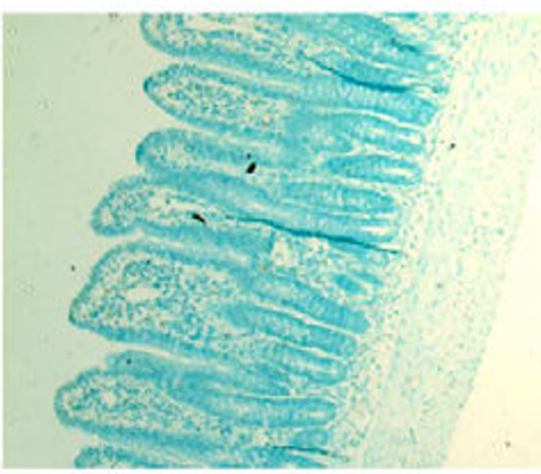

(G)

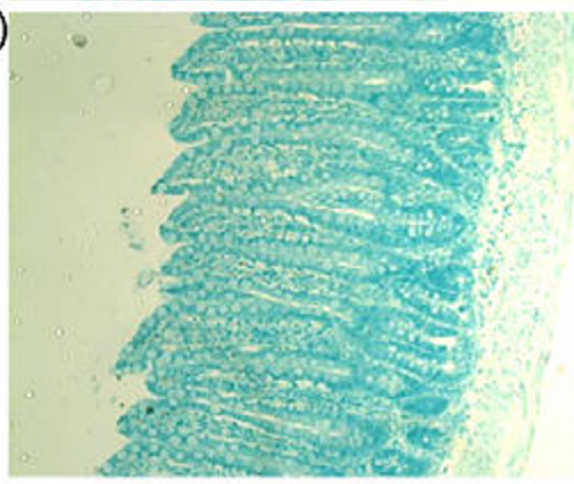

(B)

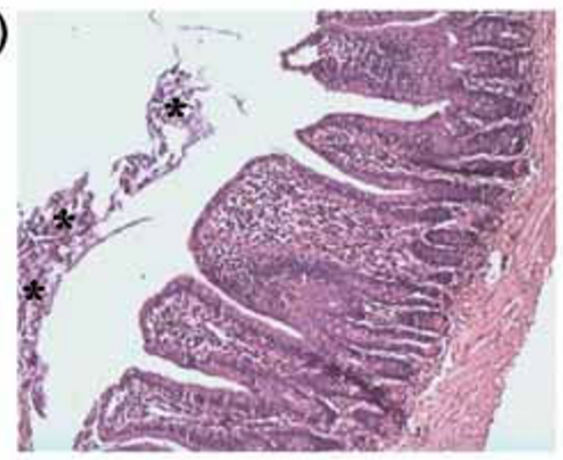

(D)

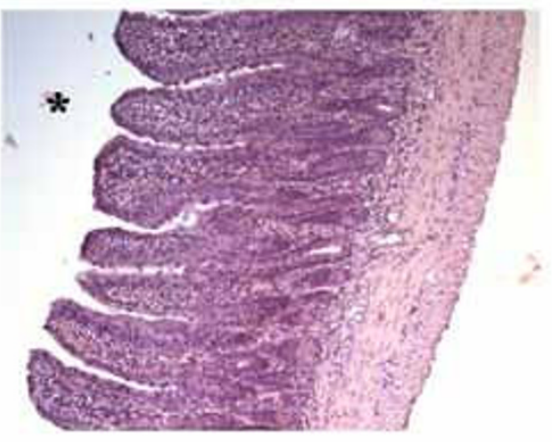

(F)

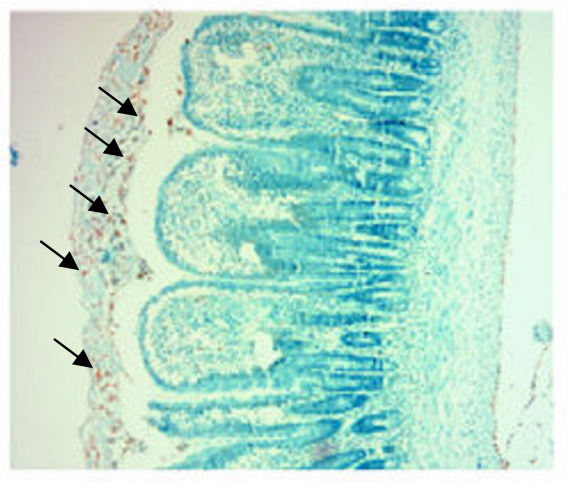

H)

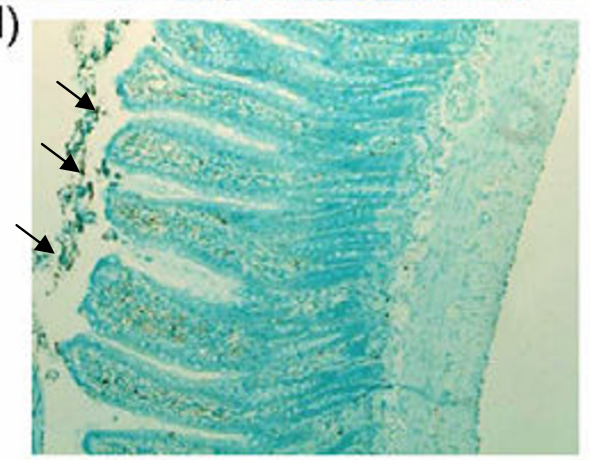

Figure 6 Histological images of the distal small intestine of sham and IO rats treated with vehicle or ML-7. (Panels A to D) Sections of intestinal tissues were stained with $\mathrm{H} \&$ E. (Panels $\mathbf{E}$ to $\mathbf{H}$ ) The apoptotic level of intestinal tissues was determined by TUNEL assay. All photomicrographs were taken at 100× magnification. Representative images shown were intestinal tissues of four groups of rats: $(\mathbf{A}, \mathbf{E}) \operatorname{sham}+\mathbf{V},(\mathbf{B}, \mathbf{F}) ! O+\mathbf{V},(\mathbf{C}, \mathbf{G})$ sham+ML-7, and (D, H) IO+ML-7. The IO-induced villous blunting, epithelial shedding (asterisk) and cell apoptosis (arrows) were partially prevented by ML-7, but not by vehicle. 
(A)

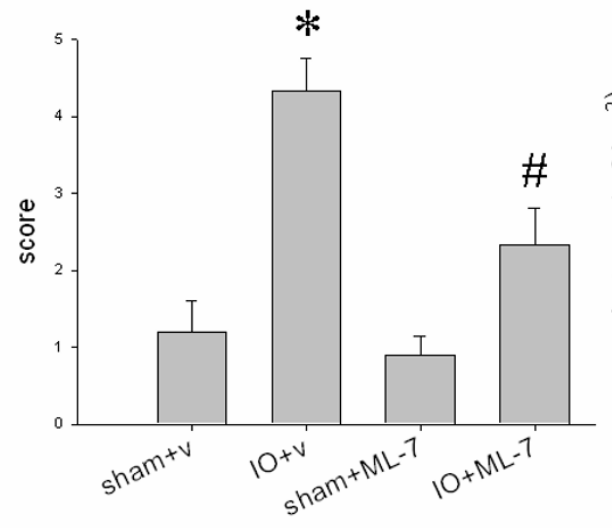

(B)

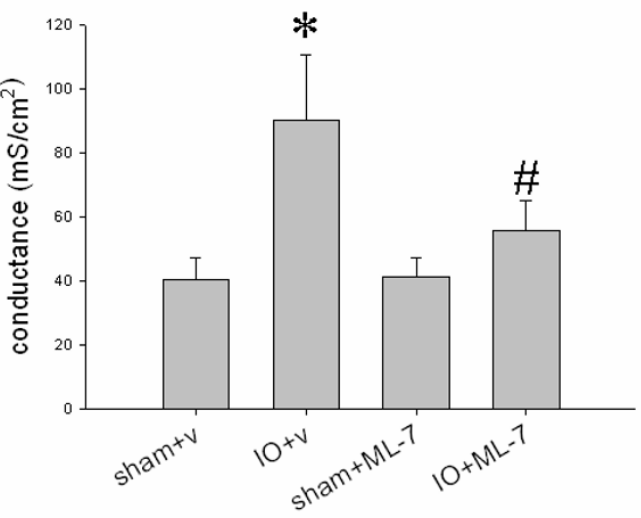

(C)

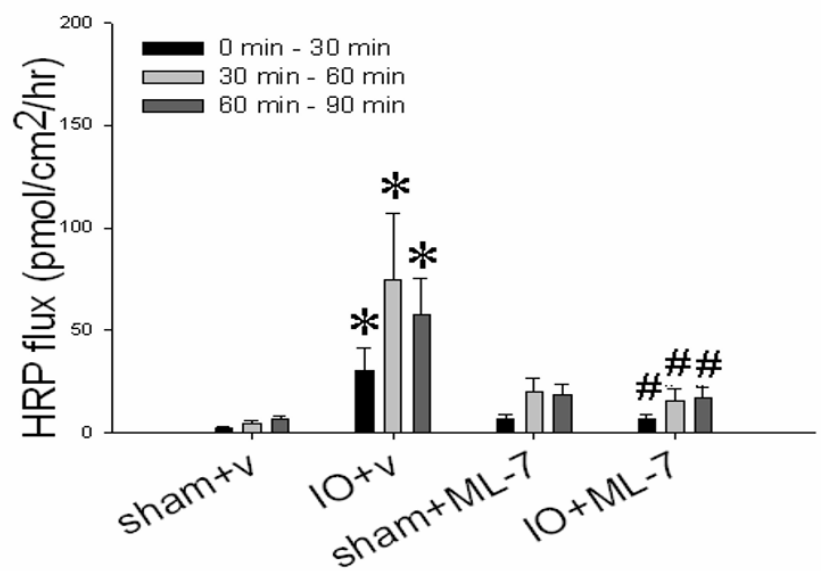

Figure 7 Administration of ML-7 reduced the histopathological score, and inhibited the epithelial permeability rise in IO rats. Intestinal tissues of sham and IO rats treated with vehicle (v) or ML-7 were graded for histological damage (A), or mounted on Ussing chambers for the measurement of electrical conductance (B) and mucosal-to-serosal HRP fluxes (C). $n=5-7 /$ group. ${ }^{*} p<0.05$ vs. sham rats. $\# p<0.05$ vs. $10+v$ rats.

(A)

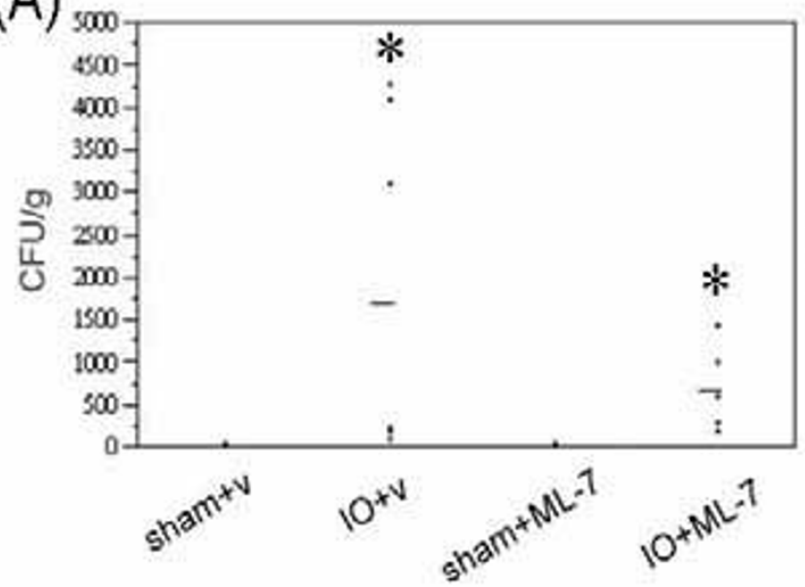

(B)

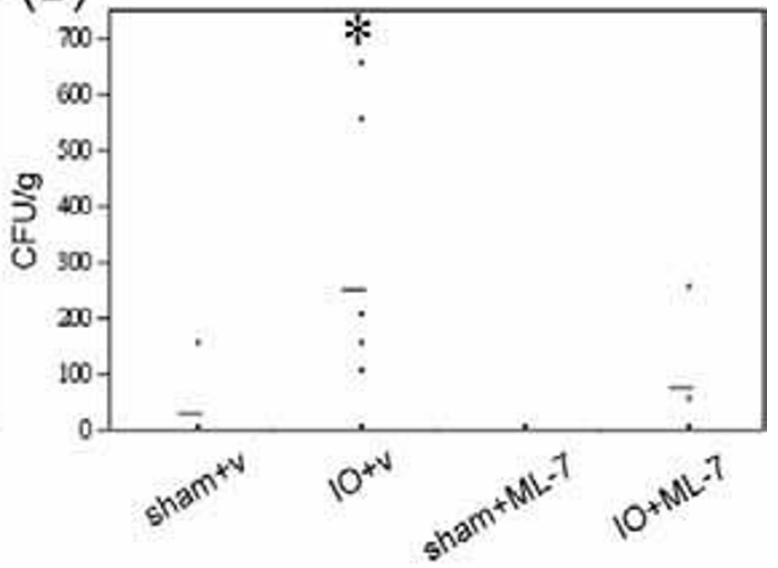

Figure 8 IO-induced augmentation of BT was not prevented by ML-7. (A) Spleen and (B) liver tissues of sham and IO rats treated with vehicle (v) or ML-7 were examined for bacterial growth. Each dot represents the bacterial CFU in the visceral organ of an individual rat. The line indicates the mean for each group. $n=5-7 /$ group. ${ }^{*} p<0.05$ vs. respective sham rats. 
gesting an MLCK-dependent increase of paracellular permeability. Recent findings indicated that epithelial MLC phosphorylation not only dilates cell-cell contacts, but also triggers the endocytosis of tight junctional proteins, such as occludin and ZO-1, into specialized apical vacuolar compartments $[37,38]$. Whether remodeling of tight junctional proteins occurs following bowel obstruction warrants further investigation.

The putative schema for crypt-villus axis involves newly proliferated enterocytes in the crypt base moving toward the villous apex, in which enterocytes mature and differentiate into tight monolayers during the migration process, and ultimately slough off at the 'extrusion zone' $[14,39,40]$. Accumulating evidence indicated that reorganization of actin filaments and phosphorylation of myosin are required for cell extrusion, and maintenance of epithelial barrier function during physiological cell turnover [14,39-43]. We noticed that the mucosal injury including epithelial shedding was ameliorated in $\mathrm{IO}+\mathrm{ML}-$ 7 rats, indicating an increase in MLCK activation was responsible for premature enterocytic sloughing after bowel obstruction. This forms the first report using an animal model that an MLCK-dependent mechanism is responsible for excessive villous epithelial shedding contributing to imbalance of the intestinal crypt-villus axis.

Lastly, treatment with ML-7 did not significantly decrease the bacterial CFU numbers in the spleen and liver, suggesting that bacterial passage through the obstructed gut may not involve MLCK-dependent paracellular pathways. Administration of ML-7 abolished IOinduced increase of tissue conductance and HRP flux, suggesting that the flux of ions and HRP was mainly through paracellular routes in obstructed guts. However, both paracellular and transcytotic routes may be responsible for the translocation of bacteria across epithelial monolayer. Previous studies demonstrated that under proinflammatory stress and metabolic energy depletion, poorly invasive bacteria were internalized into gut epithelial cells via lipid raft-mediated process and were readily transcytosed $[44,45]$. Other reports indicated that dilatation of tight junctions and activation of MLCK were associated with BT in cell culture studies and animal models of endotoxemia and stress $[15,16,46]$. It is speculated that bacterial translocation in IO models may opt for transcellular pathways, and therefore, inhibition by ML-7 did not significantly decrease bacterial influx in obstructed guts. Whether IO triggers transcellular passages of luminal bacteria is currently being studied.

In conclusion, bowel obstruction evoked morphological and functional abnormalities of the gut mucosa, and triggered BT to extraintestinal organs. Increase of the MLCK-dependent epithelial shedding and paracellular permeability were responsible for the elevated flux of macromolecular antigens in the distal small intestine.
Moreover, BT may undergo alternative routes other than paracellular passage across the epithelium.

\section{Abbreviations}

IO: intestinal obstruction; MLC: myosin light chain; MLCK: myosin light chain kinase; BT: bacterial translocation; HRP: horseradish peroxidase; CFU: colony forming unit.

\section{Competing interests}

The authors declare that they have no competing interests.

\section{Authors' contributions}

Guarantor of integrity of entire study: LCY; study concepts and design: LCY data acquisition: CCW, YZL, and LLW; data analysis/interpretation: CCW, and YZL; statistical analysis: $\mathrm{CYH}$; obtained funding: $\mathrm{CCW}$; manuscript drafting or revision for important intellectual content, literature research, manuscript editing, and manuscript final version approval: all authors.

\section{Acknowledgements}

This study is supported by a grant from the National Science Council (NSC 962320-B-002-067-MY3).

\section{Author Details}

Graduate Institute of Physiology, National Taiwan University College of Medicine, Taipei, Taiwan

Received: 23 July 2009 Accepted: 20 April 2010

Published: 20 April 2010

\section{References}

1. Cappell MS, Batke M: Mechanical obstruction of the small bowel and colon. Med Clin North Am 2008, 92:575-597.

2. Diaz JJ, Bokhari F, Mowery NT, Acosta JA, Block EF, Bromberg WJ, Collier BR, Cullinane DC, Dwyer KM, Griffen MM, Mayberry JC, Jerome R: Guidelines for management of small bowel obstruction. J Trauma 2008, 64:1651-1664.

3. Blair SL, Chu DZ, Schwarz RE: Outcome of palliative operations for malignant bowel obstruction in patients with peritoneal carcinomatosis from nongynecological cancer. Ann Surg Oncol 2001, 8:632-637

4. Sagar PM, MacFie J, Sedman P, May J, Mancey-Jones B, Johnstone D: Intestinal obstruction promotes gut translocation of bacteria. Dis Colon Rectum 1995, 38:640-644.

5. Shiomi H, Shimizu T, Endo Y, Murata S, Kurumi Y, Uji Y, Tani T: Relations among circulating monocytes, dendritic cells, and bacterial translocation in patients with intestinal obstruction. World J Surg 2007, 31:1806-1812

6. Clark JA, Coopersmith CM: Intestinal crosstalk: a new paradigm for understanding the gut as the "motor" of critical illness. Shock 2007, 28:384-393.

7. Teshima CW, Meddings JB: The measurement and clinical significance of intestinal permeability. Curr Gastroenterol Rep 2008, 10:443-449.

8. Deitch EA, Bridges WM, Ma JW, Ma L, Berg RD, Specian RD: Obstructed intestine as a reservoir for systemic infection. Am J Surg 1990, 159:394-40

9. Samel S, Keese M, Kleczka M, Lanig S, Gretz N, Hafner M, Sturm J, Post S: Microscopy of bacterial translocation during small bowel obstruction and ischemia in vivo--a new animal model. BMC Surg 2002, 2:6.

10. Samel S, Keese M, Lanig S, Kleczka M, Gretz N, Hafner M, Sturm J, Post S: Supplementation and inhibition of nitric oxide synthesis influences bacterial transit time during bacterial translocation in rats. Shock 2003, 19:378-382.

11. Ma TY, Hoa NT, Tran DD, Bui V, Pedram A, Mills S, Merryfield M: Cytochalasin B modulation of Caco-2 tight junction barrier: role of myosin light chain kinase. Am J Physiol Gastrointest Liver Physiol 2000, 279:G875-G885.

12. Turner JR, Angle JM, Black ED, Joyal JL, Sacks DB, Madara JL: PKCdependent regulation of transepithelial resistance: roles of MLC and MLC kinase. Am J Physiol Cell Physiol 1999, 277:C554-C562.

13. Zolotarevsky Y, Hecht G, Koutsouris A, Gonzalez DE, Quan C, Tom J, Mrsny RJ, Turner JR: A membrane-permeant peptide that inhibits MLC kinase 
restores barrier function in in vitro models of intestinal disease. Gastroenterology 2002, 123:163-172.

14. Bullen TF, Forrest S, Campbell F, Dodson AR, Hershman MJ, Pritchard DM Turner JR, Montrose MH, Watson AJ: Characterization of epithelial cell shedding from human small intestine. Lab Invest 2006, 86:1052-1063.

15. Ferrier L, Mazelin L, Cenac N, Desreumaux P, Janin A, Emilie D, Colombel JF, Garcia-Villar R, Fioramonti J, Bueno L: Stress-induced disruption of colonic epithelial barrier: role of interferon-gamma and myosin light chain kinase in mice. Gastroenterology 2003, 125:795-804.

16. Moriez R, Salvador-Cartier C, Theodorou V, Fioramonti J, Eutamene H, Bueno $L$ : Myosin light chain kinase is involved in lipopolysaccharideinduced disruption of colonic epithelial barrier and bacterial translocation in rats. Am J Pathol 2005, 167:1071-1079.

17. Scott KG, Meddings JB, Kirk DR, Lees-Miller SP, Buret AG: Intestinal infection with Giardia spp. reduces epithelial barrier function in a myosin light chain kinase-dependent fashion. Gastroenterology 2002, 123:1179-1190

18. Buret A, Gall DG, Olson ME: Effects of murine giardiasis on growth, intestinal morphology, and disaccharidase activity. J Parasitol 1990, 76:403-409.

19. Scott KG, Yu LC, Buret AG: Role of CD8+ and CD4+ T lymphocytes in jejunal mucosal injury during murine giardiasis. Infect Immun 2004, 72:3536-3542

20. Chiu CJ, McArdle AH, Brown R, Scott HJ, Gurd FN: Intestinal mucosal lesion in low-flow states. I. A morphological, hemodynamic, and metabolic reappraisal. Arch Surg 1970, 101:478-483.

21. Yang PC, Jury J, Soderholm JD, Sherman PM, McKay DM, Perdue MH: Chronic psychological stress in rats induces intestinal sensitization to luminal antigens. Am J Pathol 2006, 168:104-114.

22. Yu LC, Montagnac G, Yang PC, Conrad DH, Benmerah A, Perdue MH: Intestinal epithelial CD23 mediates enhanced antigen transport in allergy: evidence for novel splice forms. Am J Physiol Gastrointest Liver Physiol 2003, 285:G223-G234.

23. Hsiao JK, Huang CY, Lu YZ, Yang CY, Yu LC: Magnetic resonance imaging detects intestinal barrier dysfunction in a rat model of acute mesenteric ischemia/reperfusion injury. Invest Radiol 2009, 44:329-335.

24. Kabaroudis A, Papaziogas B, Koutelidakis I: Disruption of the small intestine mucosal barrier after intestinal occlusion: a study with light and electron microscopy. J Invest Surg 2003, 16:23-28.

25. Rana SV, Bhardwaj SB: Small intestinal bacterial overgrowth. Scand J Gastroenterol 2008, 43:1030-1037.

26. Morehouse JL, Specian RD, Stewart JJ, Berg RD: Translocation of indigenous bacteria from the gastrointestinal tract of mice after oral ricinoleic acid treatment. Gastroenterology 1986, 91:673-682.

27. Abreu MT, Palladino AA, Arnold ET, Kwon RS, McRoberts JA: Modulation of barrier function during Fas-mediated apoptosis in human intestinal epithelial cells. Gastroenterology 2000, 119:1524-1536.

28. Chin AC, Vergnolle N, MacNaughton WK, Wallace JL, Hollenberg MD, Buret AG: Proteinase-activated receptor 1 activation induces epithelial apoptosis and increases intestinal permeability. Proc Natl Acad Sci USA 2003, 100:11104-11109.

29. Yu LC, Flynn AN, Turner JR, Buret AG: SGLT-1-mediated glucose uptake protects intestinal epithelial cells against LPS-induced apoptosis and barrier defects: a novel cellular rescue mechanism? FASEB J 2005, 19:1822-1835

30. Azuara D, Sola A, Hotter G, Calatayud L, de Oca J: Apoptosis inhibition plays a greater role than necrosis inhibition in decreasing bacterial translocation in experimental intestinal transplantation. Surgery 2005 137:85-91.

31. Chin AC, Parkos CA: Neutrophil transepithelial migration and epithelia barrier function in IBD: potential targets for inhibiting neutrophil trafficking. Ann N Y Acad Sci 2006, 1072:276-287.

32. Yu LC, Huang CY, Kuo WT, Sayer H, Turner JR, Buret AG: SGLT-1-mediated glucose uptake protects human intestinal epithelial cells against Giardia duodenalis-induced apoptosis. Int J Parasitol 2008, 38:923-934

33. Bruewer M, Luegering A, Kucharzik T, Parcos CA, Madara JL, Hopkins AM, Nustat A: Proinflammatory cytokines disrupt epithelial barrier function by apoptosis-independent mechanisms. J/mmuno/ 2003, 171:6164-6172

34. Wang F, Graham WV, Wang Y, Witkowski ED, Schwarz BT, Turner JR: Interferon-gamma and tumor necrosis factor-alpha synergize to induce intestinal epithelial barrier dysfunction by up-regulating myosin light chain kinase expression. Am J Pathol 2005, 166:409-419.

35. Clayburgh DR, Rosen S, Witkowski ED, Wang F, Blair S, Dudek S, Garcia JG, Alverdy JC, Turner JR: A differentiation-dependent splice variant of myosin light chain kinase, MLCK1, regulates epithelial tight junction permeability. J Biol Chem 2004, 279:55506-55513.

36. Graham WV, Wang F, Clayburgh DR, Cheng JX, Yoon B, Wang Y, Lin A, Turner JR: Tumor necrosis factor-induced long myosin light chain kinase transcription is regulated by differentiation-dependent signaling events. Characterization of the human long myosin light chain kinase promoter. J Biol Chem 2006, 281:26205-26215.

37. Shen L, Black ED, Witkowski ED, Lencer WI, Guerriero V, Schneeberger EE, Turner JR: Myosin light chain phosphorylation regulates barrier function by remodeling tight junction structure. J Cell Sci 2006, 119:2095-2106.

38. Utech M, Ivanov Al, Samarin SN, Bruewer M, Turner JR, Mrsny RJ, Parkos CA, Nusrat A: Mechanism of IFN-gamma-induced endocytosis of tight junction proteins: myosin II-dependent vacuolarization of the apical plasma membrane. Mol Biol Cell 2005, 16:5040-5052.

39. Madara JL: Maintenance of the macromolecular barrier at cell extrusion sites in intestinal epithelium: physiological rearrangement of tight junctions. J Membr Biol 1990, 116:177-184.

40. Potten CS, Loeffler M: Stem cells: attributes, cycles, spirals, pitfalls and uncertainties. Lessons for and from the crypt. Development 1990, 110:1001-1020

41. Prahalad P, Calvo I, Waechter H, Matthews JB, Zuk A, Matlin KS: Regulation of MDCK cell-substratum adhesion by RhoA and myosin light chain kinase after ATP depletion. Am J Physiol Cell Physiol 2004, 286:C693-C707.

42. Rosenblatt J, Raff MC, Cramer LP: An epithelial cell destined for apoptosis signals its neighbors to extrude it by an actin- and myosindependent mechanism. Curr Biol 2001, 11:1847-1857.

43. Watson AJ, Chu S, Sieck L, Gerasimenko O, Bullen T, Campbell F, McKenna $\mathrm{M}$, Rose T, Montrose MH: Epithelial barrier function in vivo is sustained despite gaps in epithelial layers. Gastroenterology 2005, 129:902-912.

44. Clark E, Hoare C, Tanianis-Hughes J, Carlson GL, Warhurst G: Interferon gamma induces translocation of commensal Escherichia coli across gut epithelial cells via a lipid raft-mediated process. Gastroenterology 2005, 128:1258-1267.

45. Nazli A, Yang PC, Jury J, Howe K, Watson JL, Soderholm JD, Sherman PM, Perdue MH, MCKay DM: Epithelia under metabolic stress perceive commensal bacteria as a threat. Am J Pathol 2004, 164:947-957.

46. Soriani M, Santi I, Taddei A, Rappuoli R, Grandi G, Telford JL: Group B Streptococcus crosses human epithelial cells by a paracellular route. $J$ Infect Dis 2006, 193:241-250.

Pre-publication history

The pre-publication history for this paper can be accessed here: http://www.biomedcentral.com/1471-230X/10/39/prepub

doi: 10.1186/1471-230X-10-39

Cite this article as: Wu et al., Role of myosin light chain kinase in intestinal epithelial barrier defects in a rat model of bowel obstruction BMC Gastroenterology 2010, 10:39

\section{Submit your next manuscript to BioMed Centra and take full advantage of:}

- Convenient online submission

- Thorough peer review

- No space constraints or color figure charges

- Immediate publication on acceptance

- Inclusion in PubMed, CAS, Scopus and Google Scholar

- Research which is freely available for redistribution 